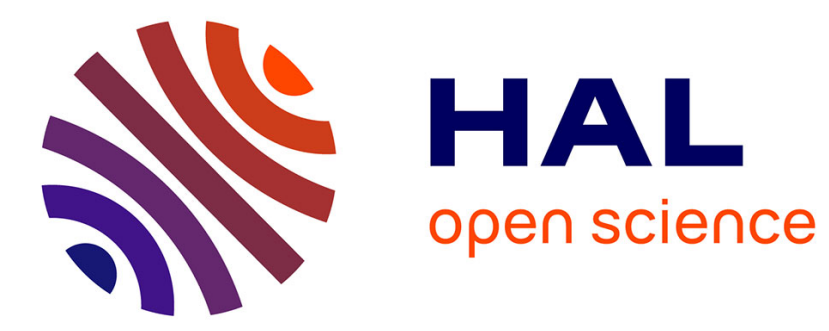

\title{
Etude comparative des modèles à une et deux exponentielles en vue d'une simulation précise des photopiles
}

\author{
J.-P. Charles, I. Mekkaoui-Alaoui, G. Bordure, P. Mialhe
}

\section{To cite this version:}

J.-P. Charles, I. Mekkaoui-Alaoui, G. Bordure, P. Mialhe. Etude comparative des modèles à une et deux exponentielles en vue d'une simulation précise des photopiles. Revue de Physique Appliquée, 1984, 19 (9), pp.851-857. 10.1051/rphysap:01984001909085100 . jpa-00245275

HAL Id: jpa-00245275

https://hal.science/jpa-00245275

Submitted on 1 Jan 1984

HAL is a multi-disciplinary open access archive for the deposit and dissemination of scientific research documents, whether they are published or not. The documents may come from teaching and research institutions in France or abroad, or from public or private research centers.
L'archive ouverte pluridisciplinaire HAL, est destinée au dépôt et à la diffusion de documents scientifiques de niveau recherche, publiés ou non, émanant des établissements d'enseignement et de recherche français ou étrangers, des laboratoires publics ou privés. 


\title{
Etude comparative des modèles à une et deux exponentielles en vue d'une simulation précise des photopiles
}

\author{
J.-P. Charles, I. Mekkaoui-Alaoui, G. Bordure \\ Centre d'Electronique de Montpellier, Université des Sciences et Techniques du Languedoc,
} Place E. Bataillon, 34060 Montpellier, France

et P. Mialhe

Ecole Normale Supérieure, B.P. 5036, Dakar-Fann, Sénégal

(Reçu le 21 octobre 1983, révisé le 16 avril 1984, accepté le 15 juin 1984)

\begin{abstract}
Résumé. - Le fonctionnement d'une photopile en tant que générateur de puissance est équivalent à celui d'un circuit électrique comportant un générateur de courant, une combinaison de résistances, et une ou deux diodes qui figurent les exponentielles des modèles. Le modèle à une exponentielle est le plus courant et est utilisé par de nombreux auteurs pour obtenir les valeurs de certains éléments par des méthodes d'approximation. Les résultats ainsi obtenus sont confrontés ici avec ceux obtenus par des méthodes d'analyse numériques précises. Un système de mesures automatiques commandẻ par ordinateur nous a permis de réaliser l'acquisition des données $I-V$ pour des conditions expérimentales variables en intensité lumineuse et en température. L'évaluation de l'efficacité des modèles à une ou à deux exponentielles permet de sélectionner celui qui est le plus approprié et d'envisager une simulation précise de la photopile en situation réelle.
\end{abstract}

\begin{abstract}
A data acquisition system including a desk computer working in real time took the current-voltage measurements under various illumination conditions. The single and double exponential models are considered here in the quadrant of the illuminated operating mode. The single diode model was mostly used to determine the values of some of its elements through approximation methods. These results are compared to those obtained through precise methods of numerical analysis. A comparative evaluation of the effectiveness of the models shows which one is best in view to a precise simulation of the solar cell.
\end{abstract}

\section{Liste des symboles.}

A Facteur de qualité de la diode $\left(A_{1}\right.$ pour D1, $A_{2}$ pour D2).

$F F=V_{\mathrm{mp}} \cdot I_{\mathrm{mp}} /\left(V_{\mathrm{oc}} \cdot I_{\mathrm{sc}}\right)$, facteur de remplissage.

I Courant dans la charge.

$I_{\mathrm{mp}} \quad$ Intensité du courant au point de puissance maximum.

$I_{\mathrm{ph}} \quad$ Intensité du photocourant.

$I_{\mathrm{s}} \quad$ Courant de saturation inverse $\left(I_{\mathrm{s} 1}\right.$ pour D1, $I_{\mathrm{s} 2}$ pour D2).

$I_{\text {sc }} \quad$ Courant de court-circuit $(V=0)$.

$k$ Constante de Boltzmann, $1,38 \times 10^{-23} \mathrm{~J} / \mathrm{K}$.

$P(V, I)=V . I$ Puissance délivrée dans la charge.

$q \quad$ Charge de l'électron, $1,602 \times 10^{-19} \mathrm{C}$.

$R(I)=-(\mathrm{d} V / \mathrm{d} I)$ Résistance dynamique au point $V-I$.

$R_{\mathrm{mp}} \quad$ Résistance dynamique au point de puissance maximum.
$R_{\mathrm{s}} \quad$ Résistance série, dans le circuit équivalent.

$R_{\mathrm{so}} \quad$ Valeur de $R(I)$ au point $: V=V_{\mathrm{oc}}, I=0$.

$R_{\mathrm{sh}} \quad$ Résistance shunt, dans le circuit équivalent.

$R_{\text {sh0 }} \quad$ Valeur de $R(I)$ au point : $V=0, I=I_{\text {sc }}$

$T \quad$ Valeur de la température en kelvin.

$V \quad$ Tension extérieure.

$V_{\text {mp }} \quad$ Valeur de la tension $V$ au point de puissance maximum.

$V_{\text {oc }} \quad$ Tension de circuit ouvert $(I=0)$.

$V_{\text {th }}=\left(A_{2} \cdot k \cdot T\right) / q$, tension thermique.

\section{Introduction.}

Une photopile se comporte, sous éclairement, comme un générateur de puissance. La caractéristique $I-V$ 
est habituellement décrite, pour un éclairement et une température donnés, par l'équation implicite :

$$
\begin{array}{r}
I=I_{\mathrm{ph}}-\frac{V+I R_{\mathrm{s}}}{R_{\mathrm{sh}}}-I_{\mathrm{s} 1}\left\{\exp \left[B_{1}\left(V+I R_{\mathrm{s}}\right)\right]-1\right\}- \\
-I_{\mathrm{s} 2}\left\{\exp \left[B_{2}\left(V+I R_{\mathrm{s}}\right)\right]-1\right\}
\end{array}
$$

$\operatorname{avec} B_{1}=q / k T, B_{2}=B_{1} / A_{2}$ et, $A_{2}=2$.

Une première représentation est donnée par le modèle à une exponentielle (Fig. 1). Dans ce modèle l'exponentielle représente le courant de diffusion de Shockley avec, idéalement, un facteur de qualité égal à l'unité. Expérimentalement, pour une bonne

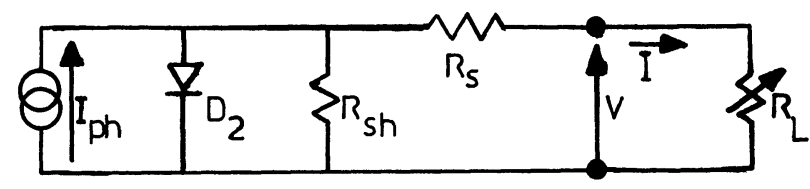

Fig. 1. - Circuit équivalent du modèle à une exponentielle ( $A_{2}$ variable) sous illumination.

[Single exponential model of the illuminated solar cell.]

description, il est nécessaire de considérer un « facteur de qualité $" A_{2}$ différent de 1 . Une description plus complète est obtenue à partir du modèle à deux exponentielles (Fig. 2). Pour ce modèle les exponentielles représentent séparément le courant de diffusion de Shockley $\left(I_{s 1}, B_{1}\right)$ et le courant dû à la recombinaison $\left(I_{\mathrm{s} 2}, B_{2}\right)$ par centres de pièges dans la zone de charge d'espace [1] avec $A_{2}$ égal à 2 .

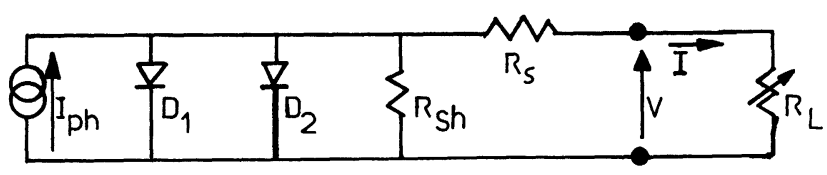

Fig. 2. - Circuit équivalent du modèle à deux exponentielles : $A_{1}=1, A_{2}$ variable, $A_{2}=2$ est un cas particulier.

[Double exponential model of the illuminated solar cell.]

De nombreuses méthodes ont été proposées [2-11] pour déterminer, moyennant des approximations, les paramètres $I_{\mathrm{ph}}, R_{\mathrm{s}}, R_{\mathrm{sh}}, I_{\mathrm{s} 2}$ et $A_{2}$. Dans toutes ces études l'équation (1) est simplifiée : l'exponentielle de $I_{\text {s1 }}$ et la résistance shunt sont omises, c'est le modèle à exponentielle simple. Des méthodes d'analyse numérique permettent de déterminer les paramètres pour le modèle à exponentielle simple [12] et pour le modèle à deux exponentielles [13] avec une grande précision.

Nous présentons ici trois programmes permettant de déterminer $R_{\mathrm{s}}, I_{\mathrm{s} 2}$ et $A_{2}$ d'après les méthodes de Singal [9], de Warashina et Ushirokawa [10] et de Araujo et Sanchez [11]. La comparaison des résultats avec ceux obtenus par analyse numérique $[12,13]$ permet de délimiter le domaine expérimental de validité des méthodes. Suit une comparaison des résultats des modèles à une ou à deux exponentielles.

\section{Données et points remarquables}

Le système d'acquisition des données comprend un APPLE II qui commande une alimentation et lit deux voltmètres par l'intermédiaire d'un BUS IEEE. La photopile étudiée est fixée sur un bloc de laiton dans lequel s'effectue une circulation d'eau. La température est mesurée par une sonde résistance de platine et un thermomètre digital. L'éclairement est fourn par deux lampes de $100 \mathrm{~W}$ quartz-halogène à filament de tungstène au travers d'un filtre à eau de $1 \mathrm{~cm}$. La puissance lumineuse des lampes est contrôlée par un circuit électronique d'asservissement optique. L’éclairement sur la photopile est réglé au moyen d'un filtre spatial à lamelles.

L'ordinateur commande la mesure de $M$ points $[V(N), I(N)]$ tels que $V(1)>V(M)$ et la visualisation de la courbe $I(V)$ en temps réel. Les données sont écrites sur disquette : référence, date, température, nombre de points et valeurs $V-I$.

Le programme de calcul des points remarquables (Fig. 3) $I_{\mathrm{sc}}$ et $R_{\mathrm{sh} 0}, V_{\mathrm{oc}}$ et $R_{\mathrm{s} 0}, I_{\mathrm{mp}}, V_{\mathrm{mp}}$ et $R_{\mathrm{mp}}$ correspond à l'algorithme suivant :

- Lecture des données sur disquette : $V(N)$, $I(N)$ pour $N$ de 1 à $M$.

- Recherche dichotomique de $N_{1}$ (valeur d'indice correspondant au changement de signe de $I$ ) à partir de $V(1)$

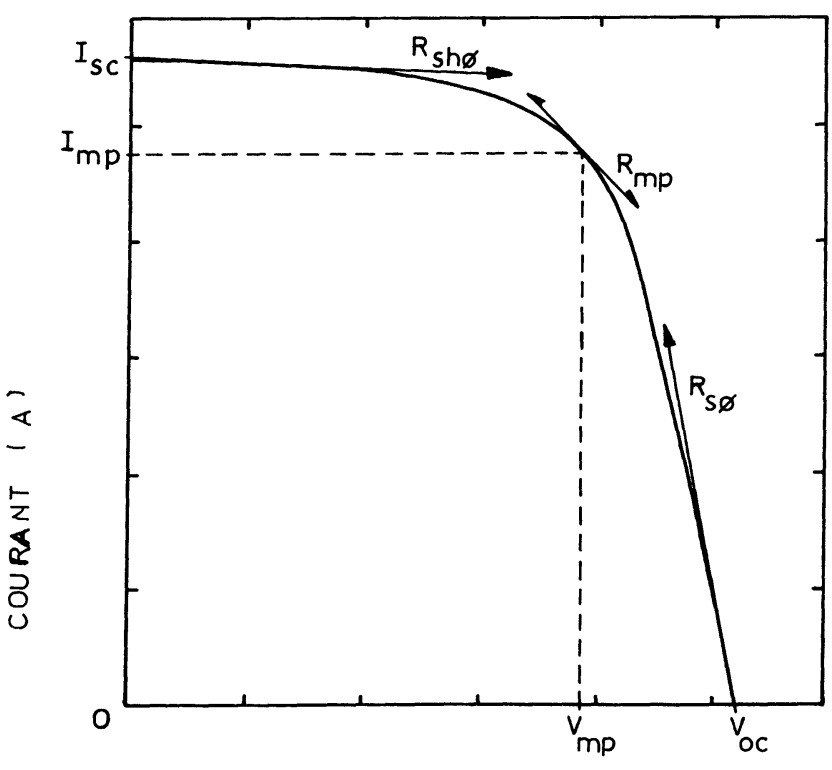

TENSION $(\mathrm{V})$

Fig. 3. - Points remarquables de la caractéristique $I(V)$ d'une photopile fonctionnant en générateur de puissance.

[Illuminated forward current-voltage characteristic. $V_{\mathrm{mp}}$ and $I_{\mathrm{mp}}$ are the experimental values at the maximum power point. $R_{\mathrm{so}}, R_{\mathrm{mp}}$ and $R_{\mathrm{sho}}$ are the particular experimental values of the dynamic resistance : $R=-(\mathrm{d} V / \mathrm{d} I)$.] 
- Calcul de la courbe de régression de $V$ sur $I$ pour 10 points autour de $N_{1}$, puis calcul de $V_{\text {oc }}$ pour $I=0$. Calcul de $R_{\mathrm{s} 0}$.

- Recherche dichotomique de $N_{2}$ (valeur d'indice correspondant au changement de signe de $V$ ), à partir de $V(M)$.

- Calcul de la courbe de régression de $I$ sur $V$ pour 10 points autour de $N_{2}$, puis calcul de $I_{\text {sc }}$ pour $V=0$. Calcul de $R_{\text {sho }}$.

- Recherche dichotomique de $N_{3}$ : valeur d'indice correspondant au changement de signe de la fonction $F=\Delta P / \Delta V$,

avec : $\Delta P=V(N) \cdot I(N)-V(N+1) \cdot I(N+1)$

et $: \Delta V=V(N)-V(N+1)$.

- Calcul de la courbe de régression de $V$ sur $F$ pour 10 points autour de $N_{3}$, puis calcul de $V_{\mathrm{mp}}$ pour $F=0$.

- Calcul de la courbe de régression de $I$ sur $V(N)-V_{m p}$ pour 10 points autour de $N_{3}$, puis calcul de $I_{\mathrm{mp}}$ pour $V(\mathrm{~N})-V_{\mathrm{mp}}=0$. Calcul de $R_{\mathrm{mp}}$.

3. Formulation mathématique des méthodes d'approximation du modèle à une exponentielle.

3.1 MÉTHOde De Singal [9]. - Dans la mesure où les conditions suivantes sont satisfaites :

- effet de $R_{\mathrm{sh}}$ négligeable,

$-\exp \left(V_{\mathrm{oc}} / V_{\mathrm{th}}\right) \gg 1$,

$-I_{\mathrm{ph}} \gg I_{\mathrm{s}}$ et $I_{\mathrm{sc}}=I_{\mathrm{ph}}$,

$-R_{\mathrm{s}} \cdot I_{\mathrm{ph}}<V_{\mathrm{oc}} / 4$,

on peut montrer à partir de l'équation (1) que :

$$
\begin{aligned}
I_{\mathrm{s}} & =I_{\mathrm{sc}} \cdot \exp \left(-V_{\mathrm{oc}} / V_{\mathrm{th}}\right) \\
\left(R_{\mathrm{s}}\right)_{F} & =V_{\mathrm{oc}} \cdot\left\{F F-(F F)_{R_{\mathrm{s}}=0}\right\} /\left(\Delta F F . I_{\mathrm{sc}}\right) \\
\left(R_{\mathrm{s}}\right)_{V} & =V_{\mathrm{oc}} \cdot\left\{V_{\mathrm{mp}} / V_{\mathrm{oc}}-\left(V_{\mathrm{mp}} / V_{\mathrm{oc}}\right)_{R_{\mathrm{s}}=0}\right\} /\left(\Delta V_{\mathrm{mp}} \cdot I_{\mathrm{sc}}\right)
\end{aligned}
$$

Les coefficients de ces trois équations étant :

$$
\begin{aligned}
& (F F)_{R_{\mathrm{s}}=0}=(1-1 / u) \cdot(1-\log u / v) \\
& \Delta F F=\left(V_{\text {oc }} / I_{\mathrm{sc}} / R_{\mathrm{s}}\right) \cdot\left\{F F-(F F)_{R_{\mathrm{s}}=0}\right\} \\
& \Delta F F=-\left\{w^{2} \cdot(u-2) / u^{3}-\right. \\
& \left.-2 . \imath \cdot 1 \cdot\left(1-\log u_{i} v\right)_{i} u^{3}\right) \\
& \left(V_{\mathrm{mp}} / V_{\mathrm{oc}}\right)_{R_{\mathrm{s}}=0}=1-\log u / v \\
& \Delta V_{\mathrm{mp}}=\left(V_{\mathrm{oc}} / I_{\mathrm{sc}} / R_{\mathrm{s}}\right) \times \\
& \times\left\{V_{\mathrm{mp}} / V_{\mathrm{oc}}-\left(V_{\mathrm{mp} /} V_{\mathrm{wc}}\right)_{R_{\mathrm{v}}=0}\right\} \\
& \Delta V_{\mathrm{mp}}=-w(u-2) / u^{2} \\
& v=V_{\mathrm{oc}} / V_{\mathrm{th}}, \quad w=v-\log v \quad \text { et } \quad u=w+1 \text {. }
\end{aligned}
$$

Notre programme utilise les données $I_{\mathrm{sc}}, V_{\mathrm{oc}}, V_{\mathrm{mp}}$ et $I_{\mathrm{mp}}$ pour résoudre les équations précédentes, c'est-àdire déterminer les valeurs des paramètres $A_{2}, R_{\mathrm{s}}$ et $I_{\mathrm{s} 2}$. L'organigramme est donné dans la figure 4 : le programme commence par une lecture des données sur disquette et le calcul des valeurs des points remar- quables. Suit une recherche dichotomique de la valeur de $A_{2}$ qui correspond à l'égalité de $\left(R_{\mathrm{s}}\right)_{F}$ avec $\left(R_{\mathrm{s}}\right)_{V}$. Les données de recherche dichotomique sont : l'intervalle de recherche ( $A 1, A 4)$, l'accroissement $A 0$ de la variable $A$, et l'erreur $E$ sur les différences relatives $\Delta R_{\mathrm{s}} /\left(R_{\mathrm{s}}\right)_{F}$ et $\Delta D / D$. Elles peuvent être modifiées par l'opérateur. Les valeurs par défaut étant :

$$
\begin{aligned}
(A 1, A 4) & =(0,3) \\
A 0 & =0,001 \\
E & =5 \% .
\end{aligned}
$$

La validité des conditions d'approximation par rapport aux résultats est ensuite testée (Tests 1 et 4 ).

3. 2 MÉthode De Warashina et UshiroKawa [10]. Cette méthode part des conditions aux limites $V_{\text {oc }}$ et $I_{\mathrm{sc}}$ dans l'équation (1). Dans la mesure où $I_{\mathrm{ph}}=I_{\mathrm{sc}}$, $\exp \left(V_{\mathrm{oc}} / V_{\mathrm{th}}\right) \gg \exp \left(R_{\mathrm{s}} \cdot I_{\mathrm{sc}} / V_{\mathrm{th}}\right)$, et où l'effet de $R_{\mathrm{sh}}$ est négligeable, on peut écrire :

$$
\begin{aligned}
& I_{\mathrm{c} 2}=I_{\mathrm{sc}} \cdot\left\{\exp \left(V_{\mathrm{oc}} / V_{\mathrm{th}}\right)-\exp \left(I_{\mathrm{sc}} \cdot R_{\mathrm{s}} / V_{\mathrm{th}}\right)\right\}^{-1} \\
& R(I)=(-\mathrm{d} V / \mathrm{d} I)=R_{\mathrm{s}}+A \cdot k \cdot T / q /\left(I_{\mathrm{sc}}-I\right)
\end{aligned}
$$

$A=A_{2}$ et $R_{\mathrm{s}}$ peuvent être alors déterminés graphiquement comme la pente et l'ordonnée à l'origine de la portion linéaire de la courbe $\left.R\left\{1 / I_{\mathrm{sc}}-I\right)\right\}$. Cette courbe se déduit de la courbe expérimentale $I(V)$.

Nous proposons ici un programme (Fig. 5) qui calcule les valeurs $R(I)$ par régression de $R$ sur $I$. La courbe $R\left\{1 /\left(I_{\mathrm{sc}}-I\right)\right\}$ est ensuite visualisée. La partie linéaire est délimitée et analysée par régression linéaire. Les valeurs de $A_{2}$ et de $R_{\mathrm{s}}$ trouvées permettent alors de déterminer $I_{\mathrm{s} 2}$ par l'équation (12).

3.3 Méthode de Araujo et Sanchez [11]. - Ces auteurs introduisent les variables réduites

$$
\begin{aligned}
& v_{\mathrm{oc}}=V_{\mathrm{oc}} / V_{\mathrm{th}} \\
& v_{\mathrm{r}}=I_{\mathrm{ph}} \cdot R_{\mathrm{s}} / V_{\mathrm{th}}
\end{aligned}
$$

$v_{\mathrm{oc}}$ et $v_{\mathrm{r}}$ sont déterminés à partir des équations fonctions du point de puissance maximum :

$$
\begin{aligned}
v_{\mathrm{oc}}= & 2 \cdot v_{\mathrm{r}}-\frac{a+1}{\log a} \cdot \log \left(1-I_{\mathrm{mp}} / I_{\mathrm{ph}}\right)-1 \\
v_{\mathrm{oc}}= & \{a \cdot \log a /(a+1)- \\
& \left.\quad-v_{\mathrm{r} \cdot}\left(1-a^{-a /(a+1)}\right)\right\} /\left(1-V_{\mathrm{mp}} / V_{\mathrm{oc}}\right)
\end{aligned}
$$

où :

$$
a=V_{\mathrm{oc}} / V_{\mathrm{th}}+1-2 \cdot R_{\mathrm{s}} \cdot I_{\mathrm{ph}} / V_{\mathrm{th}} .
$$

L'intersection des droites représentant les équations (16) et (17) donne la solution graphique $\left(v_{\mathrm{r}}, v_{\mathrm{oc}}\right)$. De là on tire $A_{2}$ (Eq. (14)) et $R_{\mathrm{s}}$ (Eq. (15)). $I_{\mathrm{s} 2}$ est calculé à partir de :

$$
I_{\mathrm{s} 2}=I_{\mathrm{ph}} \cdot \exp \left(-V_{\mathrm{oc}} / V_{\mathrm{th}}\right) .
$$




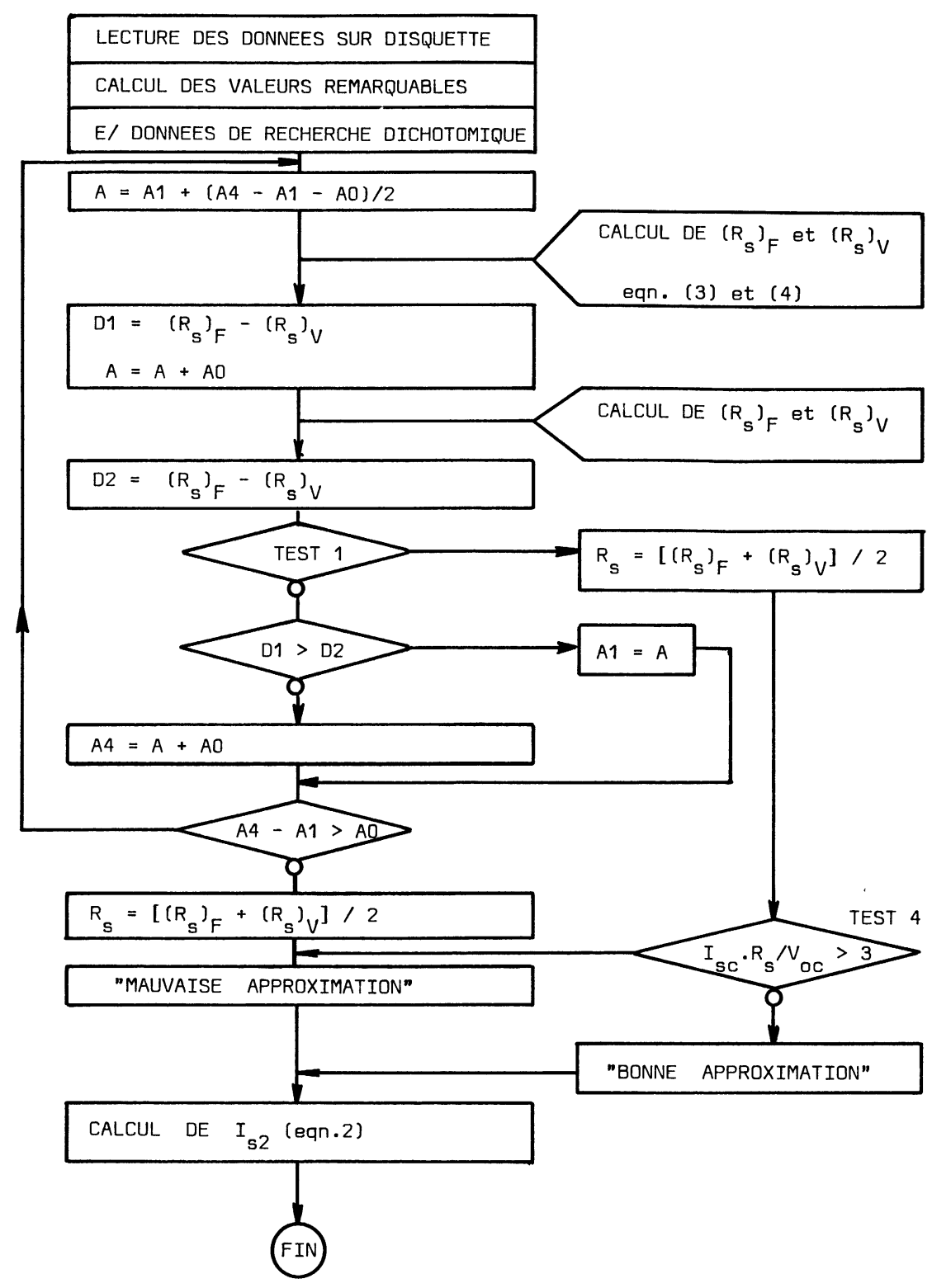

Fig. 4. - Organigramme de résolution des équations de Singal [9]. Test $1: \Delta D / D 1<E$ et $\Delta R_{\mathrm{s}} /\left(R_{\mathrm{s}}\right)_{F}<E$.

[Flow-chart of the program resolving the equations of Singal.]

Nous avons écrit un programme permettant d'arriver au résultat à partir des données : après lecture des données et calcul des points remarquables, on recherche dichotomiquement la valeur de $v_{\mathrm{r}}$ pour laquelle les équations (16) et (17) donnent le même $v_{\mathrm{oc}}$. A chaque valeur de $v_{\mathrm{r}}$ correspond une résolution des équations implicites (16) et (17) par la méthode de Regula Falsi ou par itération (résultat identique mais plus long par itération). $v_{\mathrm{oc}}$ et $v_{\mathrm{r}}$ étant connus, $A_{2}, R_{\varsigma}$ et $I_{\mathrm{s} 2}$ sont alors calculés.

\section{Résultats.}

Les résultats présentés correspondent à des mesures effectuées sur une photopile bleue au silicium, de $2 \times 2 \mathrm{~cm}$
4.1 Modèle À UNe eXPonentielle (Fig. 1). - Les variations de $A_{2}$ (Fig. 6) et de $R_{\mathrm{s}}$ (Fig. 7) sont représentées pour des mesures effectuées à température constante $\left(30^{\circ} \mathrm{C}\right)$ sous éclairement variable de $0,3 \times \mathrm{AM} 1$ jusqu'à $1,5 \times \mathrm{AM} 1$. Le résultat obtenu par la méthode de Warashina et Ushirokawa [10] se distingue nettement des autres pour les faibles éclairements. La méthode d'analyse numérique [12] donne un résultat très similaire à ceux obtenus par les méthodes d'approximations considérées $[9,11$, 13]. La variation de $R_{\mathrm{s}}$ estimée par la formule de Cape et Zehr [6] est aussi représentée (Fig. 7) à titre de comparaison :

$$
R_{\mathrm{s}}=V_{\mathrm{oc}} / I_{\mathrm{sc}}-V_{\mathrm{mp}} / I_{\mathrm{mp}}
$$




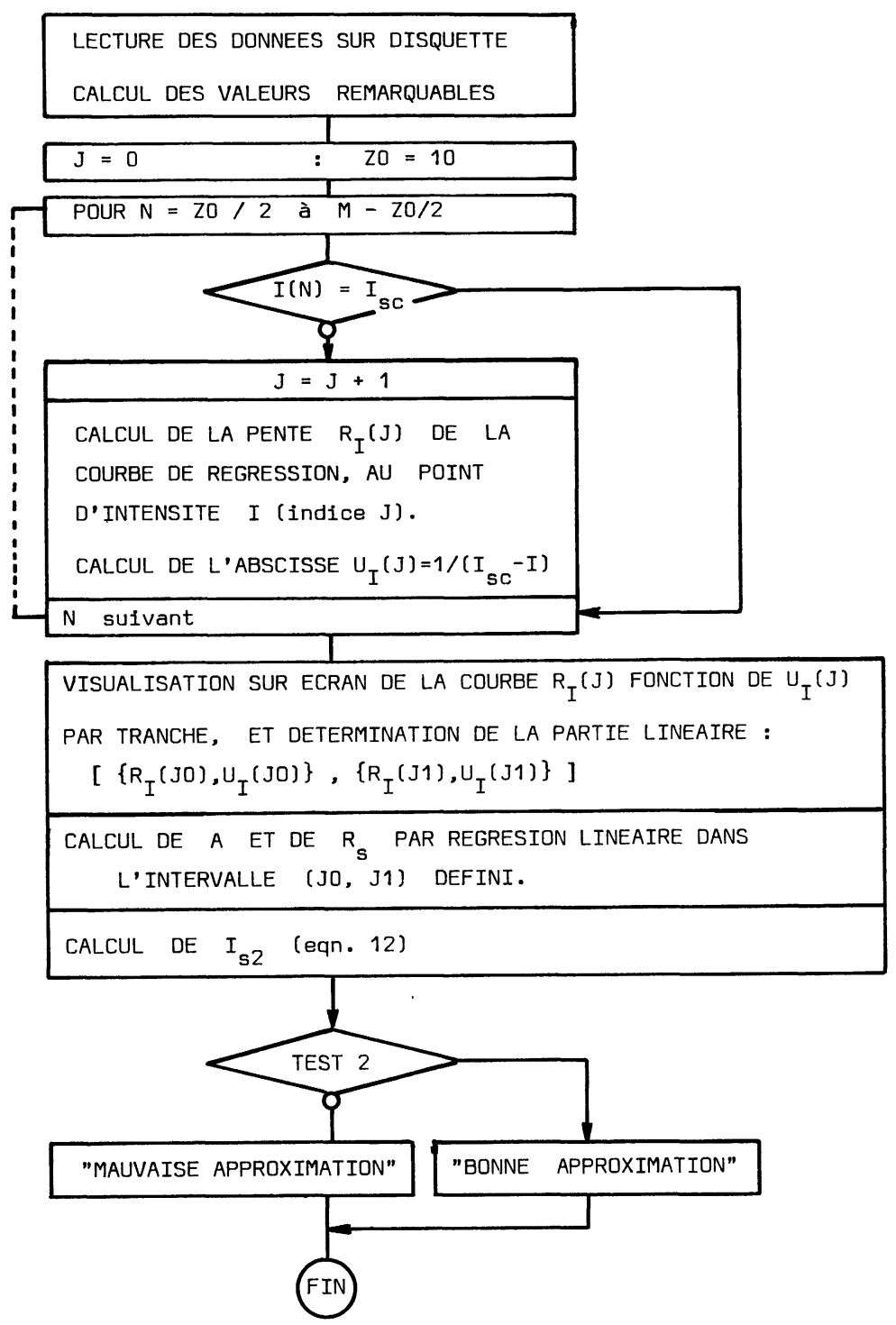

Fig. 5. - Organigramme de détermination de $R_{\mathrm{s}}, A_{2}$ et de $I_{\mathrm{s} 2}$, basé sur les équations de Warashina et Ushirokawa [10]. Test $2: \exp \left(V_{\text {oc }} / V_{\text {th }}\right) \gg \exp \left(R_{\mathrm{s}} \cdot I_{\mathrm{sc}} / V_{\mathrm{th}}\right)$.

[Flow-chart of the program giving a determination of $R_{\mathrm{s}}, A_{2}$ and $I_{\mathrm{s} 2}$ from the equations of Warashina and Ushirokawa.]

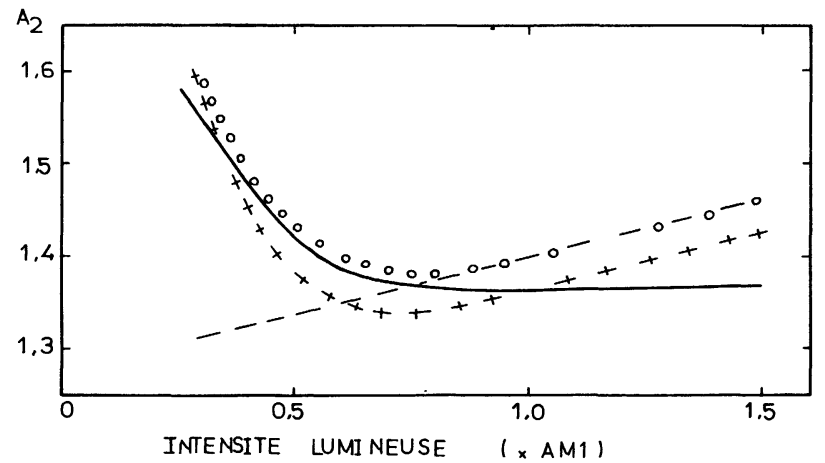

Fig. 6. - Variation de $A_{2}$ avec l'éclairement. Approximations de Singal $(++)$, de Warashina et Ushirokawa (- - ), et de Araujo et Sanchez (OO). Résultat par analyse numérique [12] en trait plein (-).

[Plots of $A_{2}$ against the illumination level. $A_{2}$ is calculated by the programs using the approximation methods [9] $(++),[10](--)$ and [11] (OO), and the numerical analysis [13] (-).]
Les valeurs ainsi obtenues diffèrent des autres dans un rapport allant jusqu'à 10 lorsque l'éclairement diminue. Cette formule basée sur trois points de mesure, et fortement influencée par $I_{\text {sc }}$, ne donnera donc un résultat valable que dans un domaine réduit d'éclairement.

Les valeurs de $I_{\mathrm{s} 2}$ calculées sont données dans le tableau I. Le courant de saturation inverse ne varie pas avec l'éclairement. La similitude des résultats de Warashina et Ushirokawa [10] avec Charles et al. [12] est à noter.

L'efficacité d'une méthode peut être évaluée selon la valeur de la moyenne quadratique des distances entre les points expérimentaux et la courbe théorique correspondant aux paramètres du circuit équivalent calculés. Cette moyenne $Q$ a été calculée pour les méthodes d'approximation [9-11] qui ne fournissent que 3 des 5 paramètres en prenant $I_{\mathrm{ph}}=I_{\mathrm{sc}}$, et $R_{\mathrm{sh}}$ infiniment grand. Ces valeurs sont portées dans le 


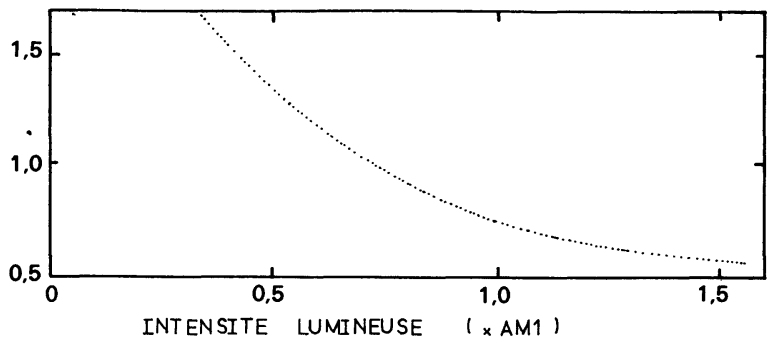

Tableau I. - Valeurs de $I_{\mathrm{s} 2}$ pour le circuit de la figure 1 avec $T=304 \pm 3 \mathrm{~K}$.

\begin{tabular}{|l|l|}
\hline $\begin{array}{c}{[9]} \\
\text { Singal }\end{array}$ & $(3,0 \pm 1,5) \cdot 10^{-8} \mathrm{~A}$ \\
\hline $\begin{array}{c}{[10]} \\
\text { Warashina } \\
\text { Ushirokawa }\end{array}$ & $(1,5 \pm 1,0) \cdot 10^{-8} \mathrm{~A}$ \\
\hline $\begin{array}{l}{[11]} \\
\text { Araujo } \\
\text { Sanchez }\end{array}$ & $(4 \pm 2) \cdot 10^{-8} \mathrm{~A}$ \\
\hline $\begin{array}{l}{[12]} \\
\text { Charles } \\
\text { Abdelkrim } \\
\text { Muoy } \\
\text { Mialhe }\end{array}$ & $(1,6 \pm 0,8) \cdot 10^{-8} \mathrm{~A}$ \\
\hline
\end{tabular}

Fig. 7. - Variation de $R_{\mathrm{s}}$ avec l'éclairement. Approximation de Cape et Zehr en pointillé (...). Les autres méthodes correspondent à la figure 6 .

[Plots of $\boldsymbol{R}_{\mathrm{s}}$ against the illumination level. (...) represents the result of the formula given in [6]. The plots are the same as in figure 6 , for the other methods.]

tableau II avec celles obtenues par analyse numérique [12]. Cette dernière méthode ne montre un avantage certain que pour les éclairements supérieurs

à $80 \%$ de $\mathrm{AM} 1$. Les valeurs relativement fortes de $Q$ à faible éclairement dénotent un certain désaccord entre l'expérience et le résultat calculé pour le modèle à une exponentielle. Cet écart peut être attribué soit au fonctionnement de la photopile elle-même, soit à la méthode de calcul. Des travaux sont en cours pour lever cette incertitude.

4.2 Modèle À DeuX eXPONEnTielles (Fig. 2). La résolution numérique de l'équation (1) à deux

Tableau II. - Moyennes quadratiques des distances entre les points expérimentaux et la courbe théorique correspondante $\left(\times 10^{-3}\right)$.

\begin{tabular}{|c|c|c|c|c|c|}
\hline & $0,3 \times \mathrm{AM} 1$ & $0,5 \times \mathrm{AM} 1$ & $0,8 \times \mathrm{AM} 1$ & AM1 & $1,5 \times$ AM1 \\
\hline $\begin{array}{c}{[9]} \\
\text { Singal }\end{array}$ & 4,9 & 2,9 & 2,3 & 1,7 & 1,9 \\
\hline $\begin{array}{c}{[10]} \\
\text { Warashina } \\
\text { Ushirokawa }\end{array}$ & 8,5 & 3,1 & 2,4 & 1,8 & 1,7 \\
\hline $\begin{array}{c}{[11]} \\
\text { Araujo } \\
\text { Sanchez }\end{array}$ & 2,9 & 2,5 & 2,1 & 1,6 & 1,8 \\
\hline $\begin{array}{c}{[12]} \\
\text { Charles } \\
\text { Abdelkrim } \\
\text { Muoy } \\
\text { Mialhe }\end{array}$ & 5,3 & 3,8 & 2,1 & 1,7 & 0,7 \\
\hline $\begin{array}{l}{[13]} \\
\text { Charles } \\
\text { Mialhe } \\
\text { Bordure }\end{array}$ & 3,8 & 2,3 & 0,8 & 0,6 & 1,2 \\
\hline
\end{tabular}


exponentielles par Charles et al. [13] permet de déterminer les 5 paramètres correspondants. La valeur obtenue pour $R_{\mathrm{s}}$ est voisine de celle obtenue précédemment et ne semble pas être influencée par l'éclairement :

$$
R_{\mathrm{s}}=0,27 \pm 0,01 \Omega .
$$

Les valeurs des courants de saturation inverse $I_{\mathrm{s} 1}$ (diode $\left.\mathrm{D} 1, A_{1}=1\right)$ et $I_{\mathrm{s} 2}$ (diode $\mathrm{D} 2, A_{2}=2$ ) sont également constantes :

$$
\begin{aligned}
& I_{\mathrm{s} 1}=(2,0 \pm 0,4) \times 10^{-11} \mathrm{~A}, \\
& I_{\mathrm{s} 2}=(4,4 \pm 0,4) \times 10^{-7} \mathrm{~A} .
\end{aligned}
$$

Les valeurs obtenues pour la moyenne quadratique $Q$ sont portées dans le tableau II pour comparaison. Ces valeurs, plus faibles que celles obtenues pour l'équation (1) à exponentielle simple, expriment une meilleure correspondance entre points expérimentaux et courbe théorique pour l'équation à deux exponentielles.

Ceci reflète le fait que le fonctionnement de la photopile met en ouvre un courant de diffusion et un courant de recombinaison dans la charge d'espace, sans prédominance réelle d'aucun de ces deux termes dans le domaine d'éclairements et à la température considérés ici.

\section{Conclusion.}

Un système d'acquisition de données commandé par ordinateur travaillant en temps réel nous a permis de traiter les mesures courant-tension pour une photopile au silicium.

Nous avons testé trois méthodes graphiques basées sur des approximations [9-11] en les transformant en méthodes numériques. Au gain en précision s'est ainsi ajoutée une plus grande facilité d'utilisation. Les paramètres ainsi calculés pour le modèle à une exponentielle ont été comparés à ceux obtenus par la résolution numérique de l'équation (1) pour $I_{\mathrm{s} 1}=0$ [12]. Ces résultats ont été aussi comparés à ceux obtenus par résolution numérique du modèle à deux exponentielles [13].

L'efficacité des diverses méthodes a été appréciée par une comparaison des moyennes quadratiques des distances entre les points expérimentaux et la courbe théorique correspondante.

Pour des éclairements supérieurs à AM1 les résultats obtenus par approximation tendent vers celui fourni par résolution numérique de l'équation à une exponentielle. A faible éclairement toutefois, les résultats des méthodes d'approximation s'éloignent notablement de ceux obtenus par la résolution numérique. La mauvaise description des mesures, dans ces conditions, peut être attribuée soit au modèle, soit aux méthodes de calculs et demande une étude approfondie. Le bon accord à fort éclairement $(1,5 \times \mathrm{AM} 1)$ avec le modèle à une exponentielle peut s'expliquer par le fait que dans ces conditions les pièges susceptibles d'intervenir dans la recombinaison sont saturés et alors le processus de diffusion devient prépondérant comme sous concentration. Ces observations doivent amener les utilisateurs de telles méthodes à être très attentifs aux conditions expérimentales et aux approximations mises en jeu.

Les résultats donnés par la résolution numérique de l'équation à deux exponentielles est en meilleur accord avec l'expérience pour une plus grande dynamique d'éclairements $(0,3$ à $1,5 \mathrm{AM} 1)$. Le modèle à deux exponentielles est donc le plus approprié pour envisager une simulation précise de la photopile silicium en situation réelle.

Bibliographie

[1] Wolf, M., Noel, G. T., StiRn, R. J., IEEE Trans. Electron. Dev. ED-24, N. 4 (1977) 419-428.

[2] Wolf, M. and RauschenbaCh, H., Advanced Energy Conversion 3 (1963) 455-479.

[3] Panayotatos, P. and Card, H. C., Proc. 13th Photovoltaic Specialists' Conf.. Washington, DC, June 5-8, 1978 (IEEE, New York) 1978, pp. 634-638.

[4] Bonnet, D., Proc. 2nd E.C. Photovoltaic Solar Energy Conf., Berlin (W.), 23-26 April, 1979, pp. 387-395.

[5] Mialhe, P. and Charette, J., Am. J. Phys. 51 (1) (1983) 68-70.

[6] Cape, J. A. and Zehr, S. W., Proc. 14th Photovoltaic Specialists' Conf., San Diego, California, Jan. 7-10, 1980 (IEEE, New York) 1980, pp. 449-452.
[7] Rajkanan, K. and Shewchun, J., Solid-State Electron. 22 (1979) 193-197.

[8] Imamura, M. S. and Portscheller, J. I., Proc. 8th Photovoltaic Specialists' Conf., Seattle, W.A., 1970 (IEEE, New York) 1970, pp. 102-107.

[9] Singal, C. M., Solar Cells 3 (1981) 163-177.

[10] Warashina, M. and Ushirokawa, A., Jpn J. Appl. Phys. 19 (1980) supplement 19-2, 179-182.

[11] Araujo, G. L. and Sanchez, Solar Cells 5 (1982) $377-$ 386.

[12] Charles, J.-P., Abdelkrim, M., Muoy, Y. H. and Mialhe, P., Solar Cells 4 (1981) 169-178.

[13] Charles, J.-P., Mialhe, P. and Bordure, G., pour publication. 